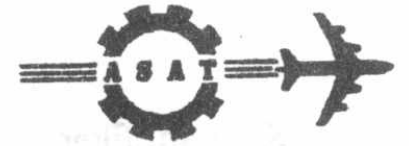

$7^{\text {th }}$ INTERNATIONAL CONF. ON AEROSPACE SCIENCES \& AVIATION TECHNOLOGY

\title{
An Adaptive Filtering Algorithm For Robot Manipulators
}

\section{T.E. Dabbous* A.Z.Badr** A.M. Wahdan** and H.S. Hashad*}

\section{Abstract}

This paper presents an adaptive filtering algorithm for robot manipulators with respect to uncertainties including unknown plant parameters (e.g. - load/tool changes, work piece variations,. ...etc.) when the robot dynamics are excited by random disturbances. Using standard variational arguments, we develop the necessary conditions for optimal identification. Based on these necessary conditions, we propose an algorithm for determining the unknowns and the corresponding estimated states. Kalman filter and an appropriate cost functional whose depending upon the states estimated and expected values form the basis of this algorithm. This algorithm is applied to two degree-of-freedom model of Unimation PUMA/560 Robot to illustrate the convergence of the parameters to its actual values during tracking of its endeffector.

\section{Introduction}

In this paper we consider the adaptive filtering problem for robot manipulator which is assumed to be governed by a nonlinear differential equation of the form [1] : $\dot{\mathrm{x}}(\mathrm{t})=H(\mathrm{x}(\mathrm{t}), \mathrm{u}(\mathrm{t}), \alpha)$

where $H$ depends on the input $\mathrm{u}$ (control variable) and the unknown parameters $\alpha$. When the effect of random loading is included in robot dynamics Kalman filter can been used to estimate the states of the robot arm. Since some of the robot arm parameters are not known, one is required to estimate first these parameters and then use Kalman filter to determine the required estimated states. We propose in this paper a certain criterion on the basis of which these unknown parameters can be identified. Using variational arguments we develop the necessary conditions for optimal identification on the basis of which the (optimal) robot parameters can be determined.

- Elect. \&Comp. Dept. Higher Tech. Inst. 10-th of Ramadan, Egypt. **Comp.\&Sys. Dept. Fac. of Eng. Ain Shams Univ. Cairo, Egypt. 
These parameters are then used in Kalman filter to determine the estimated states of robot arm.

The paper is organized as follows. In section 2, we present the mathematical nodel for robot manipulator. In section 3, we present some results which are required for developing the necessary conditions for optimal identification. Further, using variational arguments, we present the corresponding necessary conditions for optimal identification along with a proposed scheme for determining the unknowns. Finally in section 4, we present a numerical example for illustration.

\section{Problem Statement}

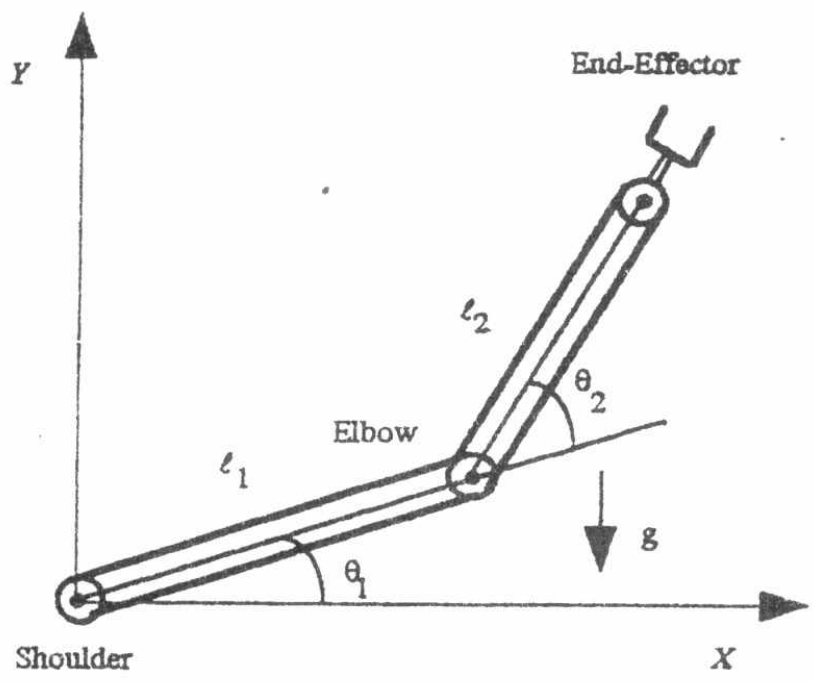

Fig. 2.1 Planar two-link mechanism.

It is known that the mechanical robot manipulator (as shown in fig. 2.1 for example) is governed by the following (nonlinear) differential equation [2]:

$\tau(t)=M(\theta(t), \alpha) \ddot{\theta}(t)+N(\theta(t), \dot{\theta}(t), \alpha)$

where:

$\tau \quad$ is $\mathrm{n} \times 1$ vector of torques or forces applied at the joint (control variable).

$\theta$ is $\mathbf{n} \times \mathbf{1}$ vector of resulting from relative joint rotations.

$\mathrm{M}$ is $\mathbf{n} \times \mathbf{n}$ inertia matrix (symmetric and positive definite).

$\mathrm{N}$ is $\mathrm{n} \times 1$ vector of centripetal and Coriolis torques.

$\alpha \quad$ is unknown system parameter.

The above nonlinear model can be linearized around the mean, and can be written as: 
$\left\{\begin{array}{cc}\dot{x}(t)=A(\alpha) x(t)+B(\alpha) u(t)+G w(t) & \\ x(0)=x_{0} & t \geq 0 .\end{array}\right.$

where $A \in \Re^{(\mathrm{axa})}, \mathrm{B} \in \mathfrak{R}^{(\mathrm{m} \times \mathrm{m})}, \mathrm{u} \in \mathfrak{R}^{\mathrm{m}}$, and $\mathrm{x}_{0}$ is a random vector with zero mean and covariance matrix $P_{0}$ and vector $w(t) ; t \geq 0$ is a Gaussian noise representing random disturbance in loading.

The observation process is assumed to be given by:

$\mathrm{Y}(\mathrm{t})=\mathrm{Hx}(\mathrm{t})+\mathrm{v}(\mathrm{t})$,

where $Y(t) \in \Re^{m}$ is a configuration vector, which is a function of the end-effector vertical position and the additional task. $H \in \Re^{(\operatorname{m\times n})}$ is a known matrix and $v(t) \in \mathfrak{R}^{\mathrm{m}}$ is a Gaussian process, independent of $\mathrm{w}$ and $\mathrm{x}_{0}$ vector, representing the measurement noise.

Consider the state and observation equations (2.2) and (2.3). Then the optimal estimate $\hat{\mathbf{x}}(t)$ satisfies the following differential equation (Kalman, Bucy[4]):-

$\left\{\begin{array}{c}\hat{\dot{x}}(t)=A \hat{x}(t)+B u(t)+K^{*}(t)[Y(t)-H \hat{x}(t)] ; t \geq 0 \\ \hat{x}(0)=\bar{x}_{0}\end{array}\right.$

where $K^{*}(t)=P(t) H^{\prime} R^{-1}$.

$P(t)=E\left\{(x-\hat{x})(x-\hat{x})^{\prime}\right\} ; t \geq 0$

denotes the covariance matrix which satisfies the following differential equation:

$\left\{\begin{array}{c}\dot{P}(t)=A P(t)+P(t) A^{\prime}-P(t) H^{\prime} R^{-1} H P(t)+G Q G^{\prime} ; t \geq 0 . \\ P(0)=P_{0}\end{array}\right.$

\section{Problem Analysis}

In this section we present necessary Lemmas and Theorems to satikfy the solutions of differential equations, and then the optimal parameter is determined.

\section{Lemma 1:}

Define $S(t) \equiv E\left\{(x(t)-\bar{x}(t))(x(t)-\bar{x}(t))^{\prime}\right\} ; t \geq 0$

and $\Gamma(t) \equiv\left\{(\hat{x}(t)-\bar{x}(t))(\hat{x}(t)-\bar{x}(t))^{\prime}\right\} ; t \geq 0$ 
where $\hat{x}(t) \equiv \hat{x}(t, \alpha)$ is the solution of (2.4) for any $\alpha$, and $\bar{x}(t) \equiv E\{x(t)\} ; t \geq 0$. The following Lemma shows that the matrix $P, S$ and $\Gamma$ satisfy certain differential equations:-

$$
\left\{\begin{array}{c}
\frac{d}{d t} S(t)=A(\alpha) S(t)+S(t) A(\alpha)^{\prime}+G^{\prime} G^{\prime} t \geq 0 \\
S(0)=P_{0}
\end{array}\right.
$$

$$
\begin{aligned}
& \text { and }
\end{aligned}
$$

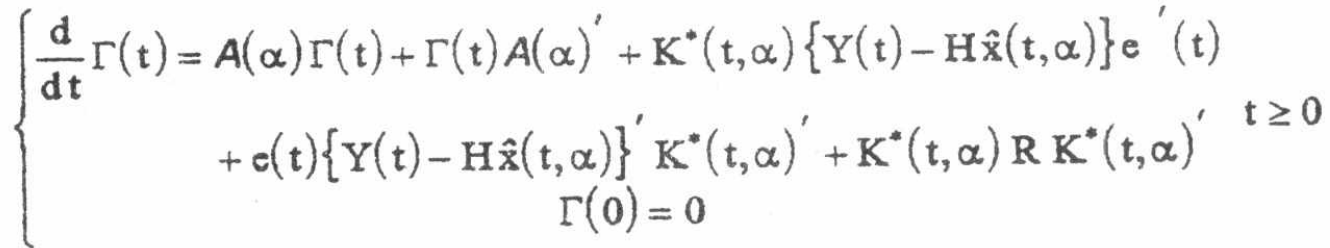

where $\mathrm{K}^{*}(\mathrm{t}, \alpha)=\mathrm{P}(\mathrm{t}, \alpha) \mathrm{H}^{\prime} \mathrm{R}^{-1}$ and for every $\alpha$, the matrix $\mathrm{P}(\mathrm{t}, \alpha) ; \mathrm{t} \geq 0$, satisfies the differential equations (2.4) and (2.6). Further, $e(t)=(\hat{x}(t)-\bar{x}(t)) ; t \geq 0$, satisfies the following differential equation [6]:

$$
\left\{\begin{array}{c}
\frac{d}{d t} e(t)=A(\alpha) e(t)+K^{*}(t, \alpha)\{Y(t)-H \hat{x}(t)\} ; t \geq 0 \\
e(0)=0
\end{array}\right.
$$

\section{Remark 1:}

$$
\text { Let } L(t, \alpha)=P(t, \alpha)-S(t, \alpha)+\Gamma(t, \alpha) \text {, }
$$

where $P, S$, and $\Gamma$ are the solutions of eq.(5.2-5), eq.(3.3) and eq.(3.4), respectively. Note that when the true parameter is introduced to the Kalman filter equation, it is clear that there is a time $t^{*}$ such that $\|P(t, \alpha)\|\left\langle\varepsilon\right.$ for all $t>t^{*}$ for any sufficiently small $\varepsilon>0$. Using this fact in equation (3.5) it follows that $e(t) \cong 0$ for all $t) t^{*}$. This implies that $\hat{x}(t) \cong \bar{x}(t)$ for all $t>t^{*}$ and in this case $L(t, \alpha) \cong 0$ for all $t) t^{*}$. However, for any other $\alpha$ the above argument does not hold and hence $L(t, \alpha) \neq 0$ for all $t$. Based on the above discussions, we may take the performance index as:

$$
J(\alpha)=\pi\left(\frac{1}{2} \int_{t_{0}}^{t_{e}}\left\{L(t, \alpha) L^{\prime}(t, \alpha)\right\} d t\right)
$$

to be our measure for estimating the unknown parameter $\alpha$ and the corresponding state.

With this set up we can now state the adaptive filtering problem as follows: 


\section{Problem 1:}

Given the driving signal $u(t) ; t \geq 0$, find an $\alpha^{0} \in a$ (parameter set) so that $\mathrm{J}\left(\alpha^{0}\right) \leq \mathrm{J}(\alpha)$ for all $\alpha \in \mathrm{a}$, where $\mathrm{J}(\alpha)$ is as given by eq.(3:7), subject. to the dynamic constraints $(2.4)-(2.6)$ and (3.3)-(3.5).

In the remaining of this section we shall develop the corresponding necessary condition for the Problem 1 and present some numerical simulation for illustration. Using (2.6), (3.3), (3.4) and (3.6), we have:

$\left\{\begin{array}{cc}\frac{d}{d t} L(t)=A L(t)+L(t) A^{\prime}+P(t) H^{\prime} R^{-1} \hat{w}(t) e^{\prime}(t)+e(t) \hat{w}^{\prime}(t) R^{-1} H P(t) \\ L(0)=0 & ; t \geq 0\end{array}\right.$

where

$\hat{w}(t)=Y(t)-H \hat{x}(t)$.

The following Lemma shows that $\hat{x}, e, P$, and Lhave Gateaux differentials that satisfy related differential equations.

We shall make use of variational arguments and the (Gateaux) differentiability of the functions on the parameter set $P$ to develop the necessary conditions for solving the problem. For that we shall need the following definitions. Let $\alpha^{8} \equiv \alpha^{0}+\varepsilon\left(\alpha-\alpha^{a}\right)$ for $\alpha^{0}, \alpha \in \mathrm{a}$ where $\alpha^{0}$ denotes the (optimal) solution to the problem 1. Since the parameter set $\mathrm{a}$ is comvex, it is clear that $\alpha^{s}$ is also an element of $\mathrm{a}$. Let $\tilde{x}(t) \equiv \lim _{\varepsilon \rightarrow 0} \frac{x^{2}(t)-x^{0}(t)}{\varepsilon} ; \quad t \geq 0$

where $\tilde{\mathrm{x}}$ denotes the (Gateaux) differential of $\mathrm{x}$ at $\alpha^{0}$ in the direction $\left(\alpha-\alpha^{0}\right)$.

\section{Lemma 2}

Consider the Problem 1 and suppose the parameter set $P$ is convex. Then $\hat{\mathrm{x}}, \mathrm{e}, \mathrm{P}$, and Lhave Gateaux differentials denoted $\tilde{\hat{\mathrm{x}}}, \tilde{\mathrm{e}}, \tilde{\mathrm{P}}$, and $\tilde{L}$, respectively, and that they satisfy the following differential equations:

$$
\begin{aligned}
& \left\{\begin{array}{c}
\frac{\mathrm{d}}{\mathrm{dt}} \tilde{\hat{\mathbf{x}}}(\mathrm{t})=\left(A^{0}-\mathrm{P}^{0} \mathrm{H}^{\prime} \mathrm{R}^{-1} \mathrm{H}\right) \tilde{\hat{\mathbf{x}}}(\mathrm{t})+\tilde{\mathrm{P}}(\mathrm{t}) \mathrm{H}^{\prime} \mathrm{R}^{-1} \hat{\mathrm{w}}^{0}+\tilde{A} \hat{\mathrm{x}}^{0} ; t \geq 0 \\
\tilde{\hat{x}}(0)=0
\end{array}\right. \\
& \left\{\begin{array}{c}
\frac{\mathrm{d}}{\mathrm{dt}} \tilde{\mathrm{e}}(\mathrm{t})=A^{0} \tilde{\mathrm{e}}(\mathrm{t})+\tilde{A} \mathrm{e}^{0}+\tilde{\mathrm{P}}(\mathrm{t}) \mathrm{H}^{\prime} \mathrm{R}^{-1} \hat{\mathrm{w}}(\mathrm{t})-\mathrm{P}^{0} \mathrm{H}^{\prime} \mathrm{R}^{-1} \mathrm{H} \tilde{\hat{\mathrm{x}}}(\mathrm{t}) ; \mathrm{t} \geq 0 \\
\tilde{\mathrm{e}}(0)=0
\end{array}\right. \\
& \left\{\begin{array}{c}
\frac{\mathrm{d}}{\mathrm{dt}} \tilde{\mathrm{P}}(\mathrm{t})=\left(A^{0}-\mathrm{P}^{0} \mathrm{H}^{\prime} \mathrm{R}^{-1} \mathrm{H}\right) \tilde{\mathrm{P}}(\mathrm{t})+\tilde{\mathrm{P}}\left(A^{0}-\mathrm{P}^{0} \mathrm{H}^{\prime} \mathrm{R}^{-1} \mathrm{H}\right)^{\prime}+\tilde{A} \mathrm{P}^{0}+\mathrm{P}^{0} \tilde{A^{\prime}} \\
\tilde{\mathrm{P}}(0)=0 \\
; t \geq 0
\end{array}\right.
\end{aligned}
$$




$$
\left\{\begin{array}{c}
\frac{\mathrm{d}}{\mathrm{dt}} \tilde{\mathrm{L}}(\mathrm{t})=\tilde{A} \mathrm{~L}^{0}+A^{0} \tilde{L}(\mathrm{t})+\mathrm{L}^{0} \tilde{A}^{\prime}+\tilde{L} A^{0}+\mathrm{Q}_{1}(\mathrm{t}) ; \mathrm{t} \geq 0 \\
\tilde{L}(0)=0
\end{array}\right.
$$

where

$$
\begin{aligned}
& \mathrm{Q}_{1}(\mathrm{t})=\tilde{\mathrm{P}} \mathrm{H}^{\prime} \mathrm{R}^{-1} \hat{\mathrm{w}}^{0} \mathrm{e}^{0^{\prime}}-\mathrm{P}^{0} \mathrm{H}^{\prime} \mathrm{R}^{-1} \mathrm{H} \tilde{\hat{\mathrm{x}}} \mathrm{e}^{0^{\prime}}+-\mathrm{P}^{0} \mathrm{H}^{\prime} \mathrm{R}^{-1} \hat{\mathrm{w}}^{0} \tilde{\mathrm{e}}(\mathrm{t})^{\prime} \\
& +\tilde{e}(t) \hat{w}^{0^{\prime}} R^{-1} H P^{0}-e^{0} \tilde{\hat{x}}^{\prime} H R^{-1} H P^{0}+e^{0} \hat{w}^{0^{\prime}} R^{-1} H \tilde{P} \text {, }
\end{aligned}
$$

where $A^{0}=A\left(t, \alpha^{0}\right), \quad \hat{w}^{0}(t)=Y(t)-H \hat{x}^{0}(t)$ and $\hat{x}^{0}, e^{0}$ and $\mathrm{P}^{0}$. are the solutions of the differential equations (3.10), (3.11) and (3.12) with $\alpha$ being replaced by $\alpha^{0}$ (optimal parameter).

Based on the above Lemma, we now present the following necessary conditions of optimality.

\section{Theorem 1: (Necessary Conditions)}

Consider the Problem 1 and suppose Lemma 1 hold. Then the optimal parameter $\alpha^{0}$ can be determined by simulations of the following set of differential equations and inequality:

$$
\begin{aligned}
& \left\{\begin{array}{c}
\frac{d}{d t} \hat{x}^{0}(t)=A\left(t, \alpha^{0}\right) \hat{x}^{0}(t)+P^{0} H R^{-1}\left[Y(t)-H \hat{x}^{0}(t)\right] ; t \geq 0 \\
\hat{x}^{0}(0)=\bar{x}(0)
\end{array}\right. \\
& \left\{\begin{array}{c}
\frac{d}{d t} e^{0}(t)=A\left(t, \alpha^{0}\right) e^{0}(t)+P^{0} H R^{-1}\left[Y(t)-H \hat{x}^{0}(t)\right] ; t \geq 0 \\
e^{0}(0)=e_{0}
\end{array}\right. \\
& \left\{\begin{array}{c}
\frac{d}{d t} P^{0}(t)=A\left(t, \alpha^{0}\right) P^{0}(t)+P^{0}(t) A^{\prime}\left(t, \alpha^{0}\right)+G Q G^{\prime}-P^{0} H R^{-1} H P^{0} ; t \geq 0 \\
P^{0}(0)=P_{0}
\end{array}\right. \\
& \int \frac{d}{d t} L^{0}(t)=A\left(t, \alpha^{0}\right) L^{0}(t)+L^{0}(t) A^{\prime}\left(t, \alpha^{0}\right)+P^{0} H R^{-1}\left[Y(t)-H \hat{x}^{0}(t)\right] e^{0^{\prime}} \\
& +\mathrm{e}^{0}\left[\mathrm{Y}(\mathrm{t})-\mathrm{H} \hat{\mathrm{x}}^{0}(\mathrm{t})\right]^{\prime} \mathrm{R}^{-1} H \mathrm{P}^{0} \\
& L^{0}(0)=L_{0} \\
& ; t \geq 0 \\
& \Psi_{2}(\mathrm{~T})=0
\end{aligned}
$$




$$
\begin{aligned}
& \left\{\begin{array}{c}
-\frac{d}{d t} \Psi_{3}(t)=\left(A\left(t, \alpha^{0}\right)-P^{0} H^{\prime} R^{-1} H\right) \Psi_{3}(t)-P^{0} H R^{-1} H \Psi_{1}(t)^{\prime} e^{0} \\
\Psi_{3}(T)=0 \quad-P^{0} H R^{-1} H \Psi_{2}(t)^{\prime} \\
; t \geq 0
\end{array} .\right.
\end{aligned}
$$

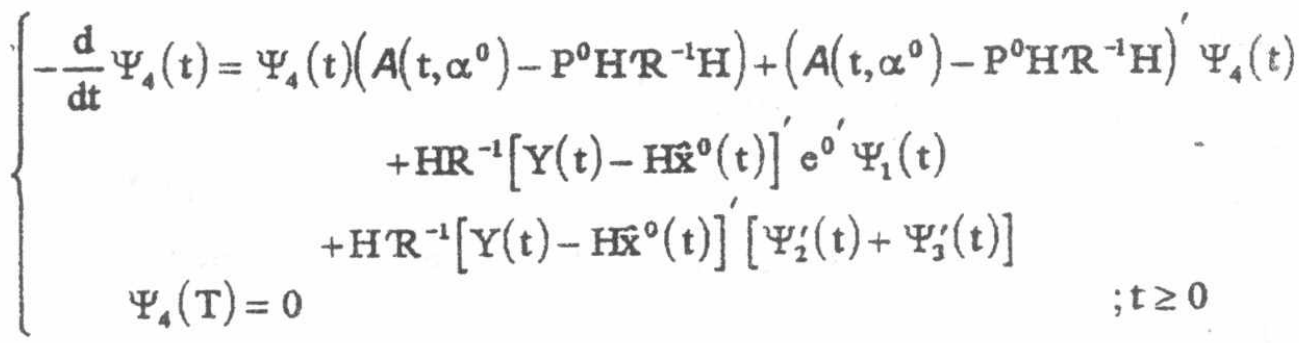

$$
\begin{aligned}
& \int_{0}^{\mathrm{T}} \mathrm{t} t\left\{\tilde{A} L^{0}(t) \Psi_{1}(t)+\tilde{A} e^{0}(t) \Psi_{2}^{\prime}(t)+2 \tilde{A} \mathrm{P}^{0}(t) \Psi_{4}(t)+\Psi_{3}(t)\left(\tilde{A} \hat{x}^{0}\right)^{\prime}\right\} d t \geq 0
\end{aligned}
$$

The proof follows from standard computations [6].

Based on the above necessary conditions, we have the following algorithm.

\section{Algorithm:}

1. Set $m=1$ and guess of $\alpha^{(m)}$.

2. Solve the differential equations (3.15)-(3.18) and gret $\hat{x}^{(m)}(t), e^{(m)}(t), P^{(m)}(t)$, and $L^{(m)}(t)$.

3. Solve the adjoint equations (3.19)- (3.22) and get $\Psi_{1}^{(m)}, \Psi_{2}^{(m)}, \Psi_{3}^{(m)}$ and $\Psi_{4}^{(m)}$.

4. Using the inequality (3.23) obtain the gradient vector $\overline{\mathbf{g}}^{(\mathbf{m})}$.

5. Update the parameter $\alpha^{(m+1)}$ using the following relation $\alpha^{(m+1)}=\alpha^{(m)}-\sigma \bar{g}^{(m)}$ where $\sigma>0$ is chosen so that $\alpha^{(m+1)} \in \mathrm{a}$ and $\mathrm{J}\left(\alpha^{(\mathrm{m}+1)}\right) \leq \mathrm{J}\left(\alpha^{(\mathrm{m})}\right)$.

6. If $\left|\mathrm{J}(\alpha)^{(\mathrm{m}+1)}-J(\alpha)^{(\mathrm{m})}\right| \leq \delta, \quad \delta(>0)$ is sufficiently sinall then stop, otherwise, set $m=m+1$ and $\alpha^{(m)} \rightarrow \alpha^{(m+1)}$ go to step 2 .

\section{Application}

For convenience we choose the UNIMATION PUMA 560 ROBOT for application as a planer mechanism moves in $x-y$ plane with two degrees of freedom as shown in fig. 2.1. Using equation (2.1) and setting $n=2$, then the nonlinear ditferential dynamical equations of motion that relate the joint torques $\left(\tau_{1}, \tau_{2}\right)$ to the angles 
$\left(\theta_{1}, \theta_{2}\right)$, the velocities $\left(\dot{\theta}_{1}, \dot{\theta}_{2}\right)$ and accelerations $\left(\ddot{\theta}_{1}, \ddot{\theta}_{2}\right)$ of the joints are given by [3] :

$$
\begin{aligned}
\tau_{1}= & \left(a_{1}+a_{2} \cos \theta_{2}\right) \ddot{\theta}_{1}+\left(a_{3}+\left(\frac{a_{2}}{2}\right) \cos \theta_{2}\right) \ddot{\theta}_{2} \\
& -\left(\left(a_{2} \sin \theta_{2}\right)\left(\dot{\theta}_{1} \dot{\theta}_{2}+\frac{\dot{\theta}_{2}^{2}}{2}\right)\right)+\left(a_{4} \cos \theta_{1}+a_{5} \cos \left(\theta_{1}+\theta_{2}\right)\right) \\
\tau_{2}= & \left(a_{3}+\left(\frac{a_{2}}{2}\right) \cos \theta_{2}\right) \ddot{\theta}_{1}+\left[a_{3}\right] \ddot{\theta}_{2}+\left(\left(a_{2} \sin \theta_{2}\right) \frac{\dot{\theta}_{1}^{2}}{2}\right) \\
& +\left(a_{5} \cos \left(\theta_{1}+\theta_{2}\right)\right)
\end{aligned}
$$

where $\left[a_{i} 1 \leq i \leq 5\right]$ are constant parameters which depend on the masses $\left\{m_{1}, m_{2}\right\}$ and the lengths $\left\{\ell_{1}, \ell_{2}\right\}$ of the robot links.

Let $y(t) \in \Re$ denote the vertical coordinate of the end-effetor and, $y(t) ; t \geq 0$ is related to the joint angles $\theta_{1}(t)$ and $\theta_{2}(t)$ by the following forward kinematic equation (output equation):-

$y(t)=\ell_{1} \sin \left(\theta_{1}(t)\right)+\ell_{2} \sin \left(\theta_{1}(t)+\theta_{2}(t)\right)$.

The joint angle $\theta_{1}(t)$ is assumed to be held at $\theta_{1}(t)=45^{\circ}$ (additional task), and $\dot{\theta}_{1}(t)=0$ at all time.

For the UNIMATION PUMA 560 ROBOT, the following parameters are given by [3], $\mathrm{m}_{1}=5.91 \mathrm{~kg} ., \mathrm{m}_{2}=11.36 \mathrm{~kg}, \ell_{1}=\ell_{2}=0.432$ meter, $a_{1}=3.82, a_{2}=2.12, a_{3}=$ $0.71, a_{4}=81.82$, and $a_{5}=24.06$.

Using Taylor series we make linearization for the nonlinear equations (4.1) and (4.2) and evaluating by the values of parameter $a$ and the nominals, the matrices $A, B$ and $\mathrm{H}$ of eq. (2.2) and (2.3) are:

$$
A=\left(\begin{array}{cccc}
0 & 1 & 0 & 0 \\
25.7536 & 0 & -1.1308 & 0 \\
0 & 0 & 0 & 1 \\
-51.3647 & 0 & 18.4562 & 0
\end{array}\right), \quad B=\left(\begin{array}{cc}
0 & 0 \\
0.71 & -1.5783 \\
0 & 0 \\
-1.5783 & 5.5566
\end{array}\right) \text {, }
$$

and

$$
H=\left(\begin{array}{cccc}
0.7309 & 0 & 0.4254 & 0 \\
0 & 0 & 1 & 0
\end{array}\right)
$$

\section{Simulated Example:}

The initial time $t_{0}=0$ sec., the final time $t_{f}=2 \mathrm{sec}$.and the no. of time interval is 100 . The adaptive filtering algorithm is applied to linearized model of UNIMATION PUMA 560 ROBOT. The numerical simulation given in Figures 
$[(4.1)-(4.5)]$ show the behavior of the actual and estimated states, $P, S, \Gamma$ and $L$, respectively. These results are taken when the initial values of the second row elements of matrix $A$ of unknown parameters are $\left(\begin{array}{lllll}40 & 1 & 1 & 1\end{array}\right)$. From these results it clear that although some of the robot parameters are not known, we have been able, through the proposed algorithm, to obtain a reasonable estimates for the states and for the unknown parameters. Note that in some cases the estimated parameter is quite different from the actual (undertying) parameter (see Figure (4.6) of the first element of $A$ ). This deviation in parameter estimate is not of great imporfance as long as the estimated states follow closely the actual states (i.e., the estimated error is small).

\section{Conclusion}

In this paper we have considered the adaptive filtering problem robot manipulator which some o the parameters are not known exactly. We have proposed a suitable criterion on the basis of which these unknowns can be determined. This criterion depends on Kalman filter estimates and the observation process. Using variational arguments we have present the necessary conditions for optimal identification. Based on these necessary conditions we have proposed an iterative scheme for determining the unknowns and the estimated states of the robot arm. The numerical results showed that the proposed scheme produces estimates which is very close to the true states. Further, the results also show the converges of the estimated parameter to the true undertying parameter.

\section{References}

1-Martin Corless, "Trajectory controllers for Uncertain Systems : Application to a Manutic R3 robot, "J. of Dynamic Systems, Measurements and Control, Dec. 1989. 2-Fu, Gonzalez and Lee,"Robotics :controh, sensing, vision and intelligence", Mc Graw-Hill, Inc., New York, 1987.

3-H. Seraji,"Configuration control of redundant manipulators: theory and implementation," IEEE Trans. on Robotics and Automation, Vol.5, No.4, 1989.

4-R. G. Brown and P. Y. C. Hwang, "Introduction to Random Signals and A Applied Kalman Filtering," John Wiley \& Sons, Inc. New York, 1992.

5-N.V.Ahmed, "Elements of finite-ddimensional systems and control theory," Longman, Scientific and Technical, London, U.K., 1988.

6-H.S.Hashad, "Optimal control in Nonlinear robot Systems, "Ph.D Thesis, AinShams University, Cairo, Egypt, 1996. 


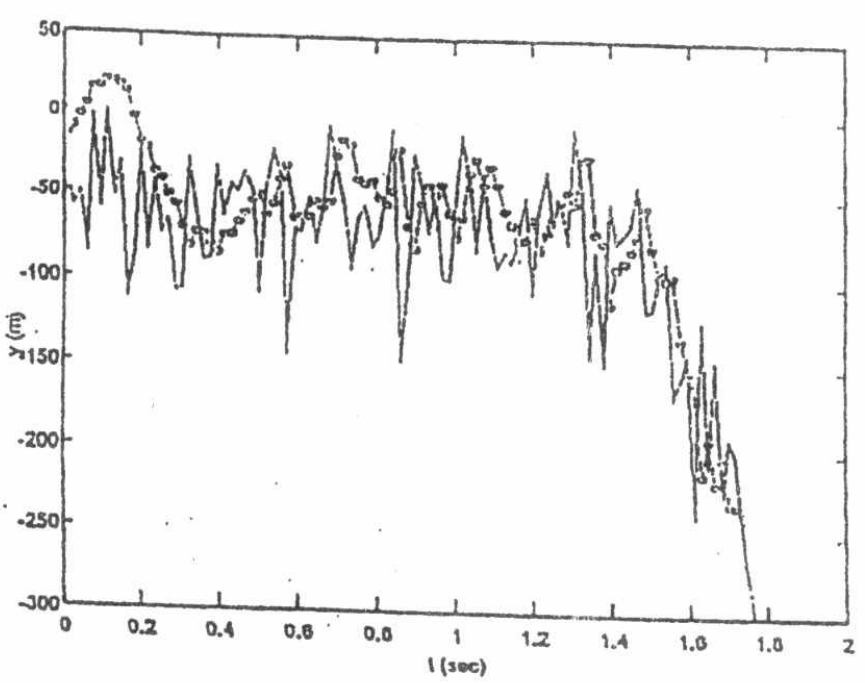

Fig. 4.1
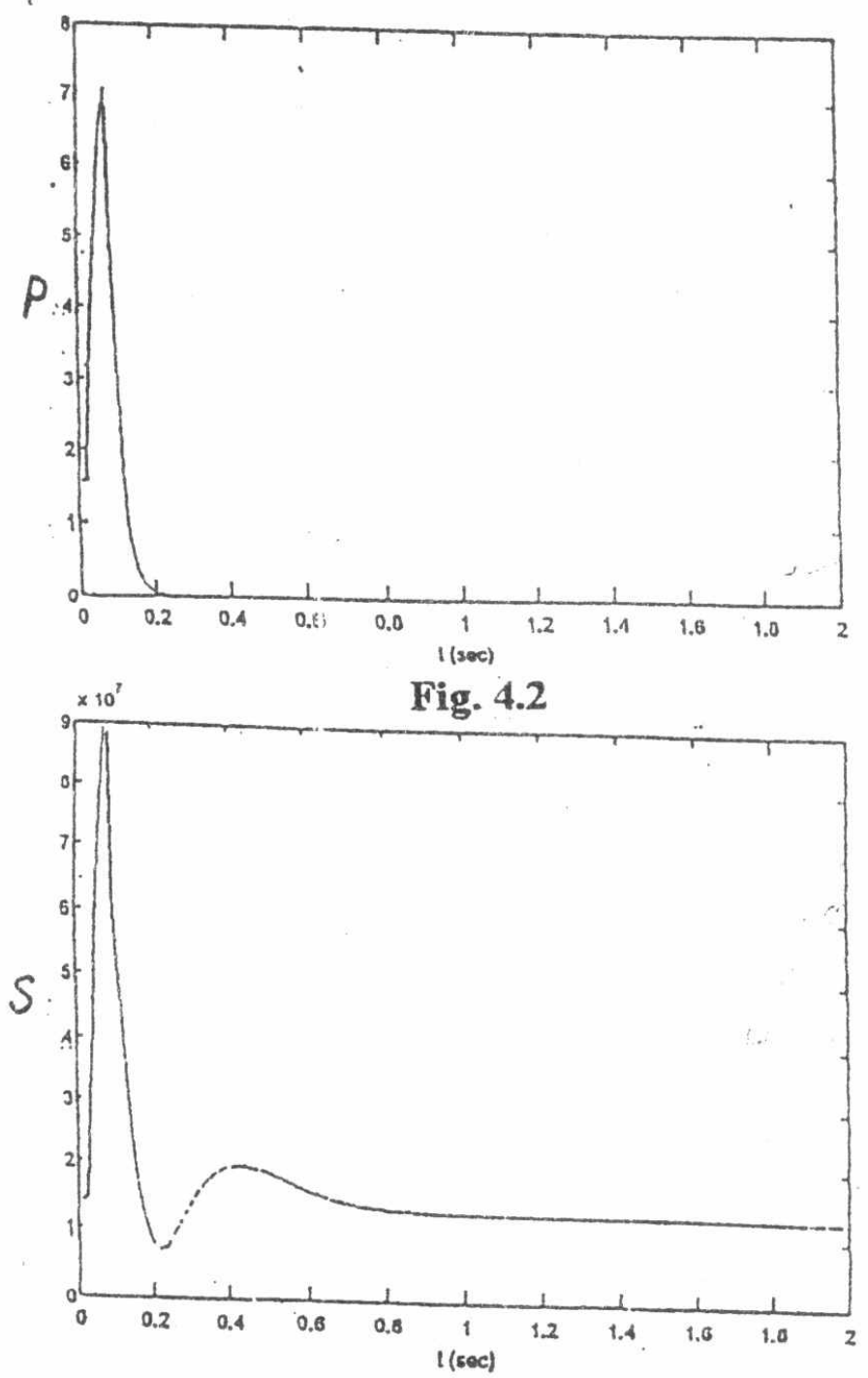

Fig. 4.3

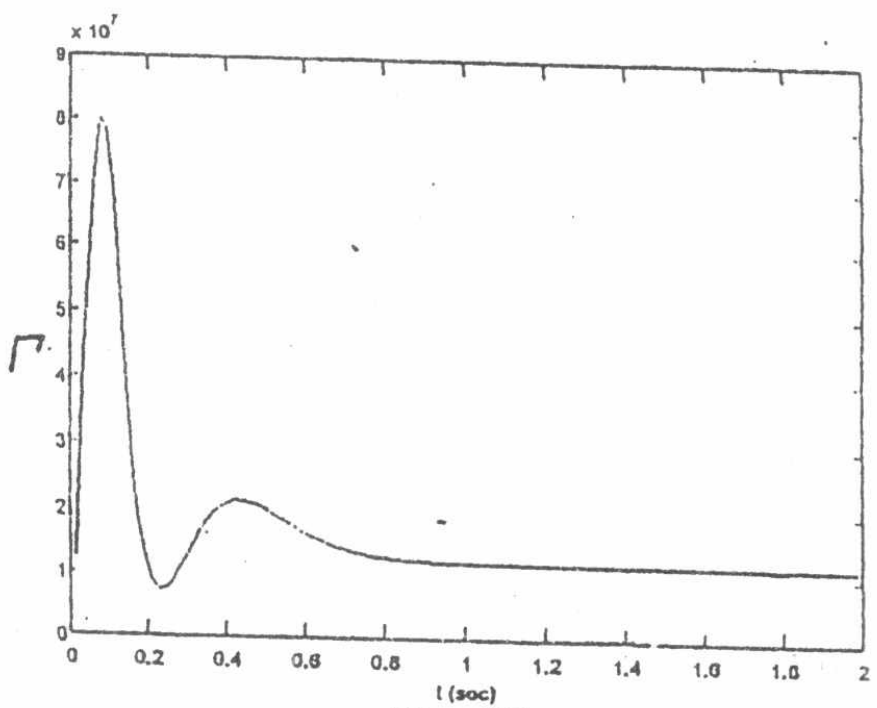

Fig. 4:4

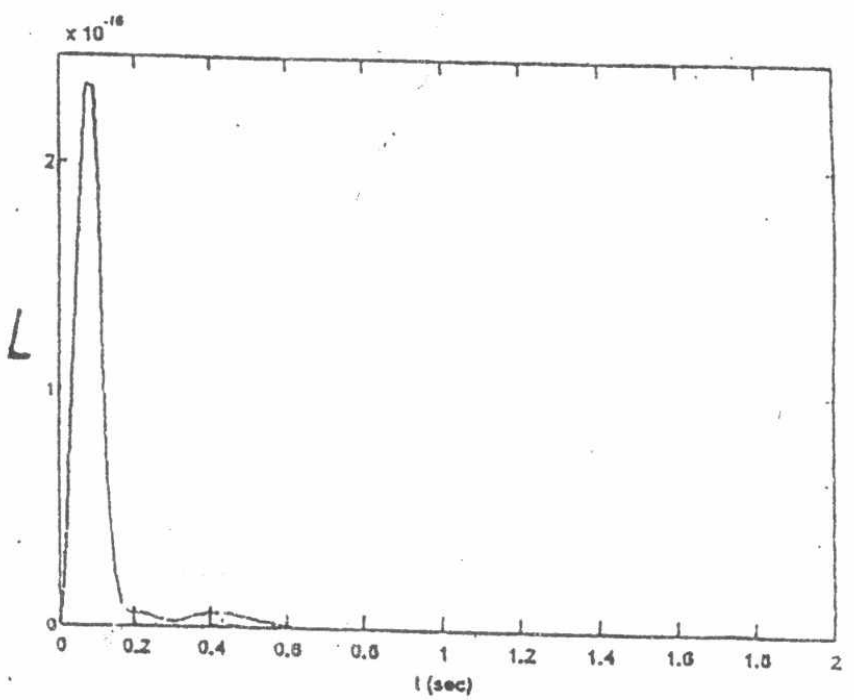

Fig. 4.5

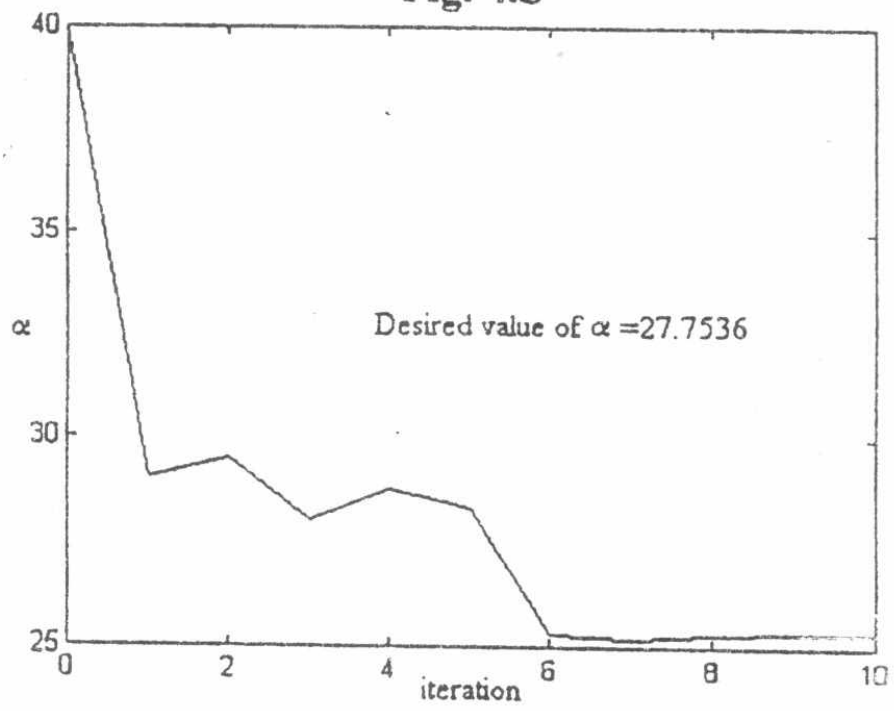

Fig. 4.6 